\title{
A bifurcation problem for a class of periodically perturbed autonomous parabolic equations
}

\author{
Mikhail Kamenskii ${ }^{1}$, Boris Mikhaylenko' and Paolo Nistri ${ }^{*}$
}

${ }^{*}$ Correspondence: pnistri@dii.unisi.it
${ }^{2}$ Dipartimento di Ingegneria
dell'Informazione e Scienze
Matematiche, Università di Siena,
Siena, 53100, Italy
Full list of author information is
available at the end of the article

\begin{abstract}
The paper deals with the problem of the existence of a branch of T-periodic solutions originating from the isolated limit cycle of an autonomous parabolic equation in a Banach space when it is perturbed by a nonlinear $T$-periodic term of small amplitude.

We solve this problem by first introducing a novel integral operator, whose fixed points are $T$-periodic solutions of the considered equation and vice versa. Then we compute the Malkin bifurcation function associated to this integral operator and we provide conditions under which the well-known assumption of the existence of a simple zero of the Malkin bifurcation function guarantees the existence of the branch. MSC: 35K58; 35B10; 35B20; 35B32
\end{abstract}

Keywords: autonomous parabolic equations; periodic perturbations; limit cycle; bifurcation; periodic solutions

\section{Introduction}

In recent years, bifurcation problems for smooth and nonsmooth dynamical systems have received a renewed attention and interest from different fields of engineering, physics and mathematics. We mention here, among others, the monographs [1-5] and the review papers $[6,7]$. Of particular interest is the study of the bifurcation of periodic solutions for periodically perturbed autonomous systems of the form:

$$
\dot{x}=\phi(x)+\varepsilon \psi(t, x, \varepsilon),
$$

where $\phi \in C^{2}\left(\mathbb{R}^{n}, \mathbb{R}^{n}\right), \psi \in C^{1}\left(\mathbb{R} \times \mathbb{R}^{n} \times[0,1], \mathbb{R}^{n}\right), \psi$ is $T$-periodic with respect to time and $\varepsilon \geq 0$ is a small parameter. Precisely, one seeks for the existence of a family of $T$-periodic solutions originating from a limit cycle $x_{0}$ of the autonomous unperturbed system.

Existence, uniqueness and asymptotic stability of bifurcating periodic solutions for system (1) are classical problems; see [8, 9]. The main tool employed in these papers is the so-called Malkin bifurcation function:

$$
f_{0}(\theta)=\int_{0}^{T}\left\langle z_{0}(\tau), \psi\left(\tau-\theta, x_{0}(\tau), 0\right)\right\rangle d \tau,
$$


where $z_{0}$ is a $T$-periodic solution of

$$
\dot{z}=-\left(\phi^{\prime}\left(x_{0}(t)\right)\right)^{*} z
$$

the adjoint system of the linearized system

$$
\dot{y}=\phi^{\prime}\left(x_{0}(t)\right) y \text {. }
$$

It is assumed that the linearized system has only one characteristic multiplier with absolute value 1 .

Since the pioneering papers $[8,9]$, a relevant bibliography devoted to this subject has been developed. From this bibliography, we quote in the sequel some of the papers more related to the present paper. In [10], the case when the cycle $x_{0}$ is not isolated was considered. By means of suitably defined bifurcation functions $f_{m, n}, m, n \in \mathbb{N}$, called Melnikov subharmonic functions, the existence of periodic solutions near to $x_{0}$ was proved. The periods of the solutions are in $m: n$ ratio with respect to the period of the perturbation term. The case when 1 is not a simple multiplier of the linearized system was treated in [11]. The existence of at least two branches of $T$-periodic solutions originating from $x_{0}$ is shown in $[12,13]$ and their stability, in the sense of Lyapunov, follows from the results of $[14,15]$. Developments of the Malkin's and Melnikov's approaches have permitted to prove several results about the existence of bifurcating solutions in [16-20]. Furthermore, the use of a Melnikov function permits to detect chaotic behavior of a suitable iterate of the Poincaré map $\Pi_{\varepsilon}$ associated to the differential equation (1), which is assumed to have a homoclinic orbit. Indeed, the existence of a simple zero of the considered Melnikov function ensures such a chaotic behavior; see [21, 22].

Very recently, in [23], a new method to prove bifurcation of a branch of asymptotically stable periodic solutions to (1) has been proposed. The method consists first in converting the problem of finding fixed points of the singular Poincaré map $\Pi_{\varepsilon}, \varepsilon \geq 0$, associated to (1) into the problem of the existence of zeros of an equation of the form:

$$
P(x)+\varepsilon Q(x, \varepsilon)=0,
$$

where $P: \mathbb{R}^{n} \rightarrow \mathbb{R}^{n}$ and $Q: \mathbb{R}^{n} \times[0,1] \rightarrow \mathbb{R}^{n}$ are given by $P(x)=\Pi_{0}(x)-x$ and $Q(x, \varepsilon)=$ $\frac{\Pi_{\varepsilon}(x)-\Pi_{0}(x)}{\varepsilon}$ with singular $P^{\prime}\left(x_{0}\right)$. Then, by a convenient scaling of the variable $x$, we introduce an equivalent equation $\Psi(w, \varepsilon)=0$. For this equation, under the usual assumption of the existence of a simple zero of the Malkin bifurcation function associated to (1), the classical implicit function theorem can be applied to prove the existence of a branch of solutions originating from $x_{0}$.

The same approach has been employed in [24] for a class of systems for which the resulting operators $P$ and $Q$ satisfy regularity conditions, which permit to apply the implicit function theorem, only along certain directions at the point $x_{0}(\cdot)$ of the limit cycle. Conditions to ensure the existence of several branches of $T$-periodic solution emanating from $x_{0}$ are provided by means of suitably defined Malkin bifurcation functions.

In all the papers cited before, the existence of periodic solutions for $\varepsilon \geq 0$ small is a consequence of the application of a convenient version of the implicit function theorem. This requires, as assumed for system (1), that $\phi \in C^{2}\left(\mathbb{R}^{n}, \mathbb{R}^{n}\right)$ and $\psi \in C^{1}\left(\mathbb{R} \times \mathbb{R}^{n} \times[0,1], \mathbb{R}^{n}\right)$. 
Under less restrictive regularity conditions, by using topological tools such as the coincidence degree [25], the Leray-Schauder degree and the related continuation principles [26-28], existence results for $\varepsilon \geq 0$ small have been proved in [29] when the autonomous system is Hamiltonian and in [30,31] when the limit cycle is isolated. More precisely, in $[29,31]$ the existence of two branches of $T$-periodic solutions was proved. Roughly speaking, in these papers, the bifurcation functions are employed to guarantee that the topological degree of certain operators is different from zero, rather than for the application of an implicit function theorem.

Topological degree arguments have been also employed in [32] to show the existence of periodic solutions for $\varepsilon \geq 0$ small in the case when the unperturbed system is nonautonomous and the perturbation consists of two nonlinear periodic terms with multiplicative different powers of $\varepsilon \geq 0$. Finally, the behavior of the bifurcating periodic solutions when the perturbation vanishes has been studied in [33] for a nonsmooth system of the form (1) having an isolated limit cycle and in [34] for nonsmooth planar Hamiltonian systems.

A first attempt to extend to infinite dimensional bifurcation problem the approach outlined in [23] has been presented in [35], with the aim of studying the bifurcation of periodic solutions for a functional differential equation of neutral type. In the present paper, we precise and generalize the idea of how to use a suitable abstract Malkin bifurcation function to deal with infinite dimensional bifurcation problems. To this aim, we consider the following autonomous differential equation of parabolic type periodically perturbed by a nonlinear term of small amplitude:

$$
\dot{x}=A x+\phi(x)+\varepsilon \psi(t, x, \varepsilon), \quad t \geq 0, \varepsilon>0,
$$

where $A$ is the infinitesimal generator of a strongly continuous semigroup $e^{A t}, t \geq 0$, acting in the Banach space $E$, satisfying the Radon-Nikodym property, $\phi: E \rightarrow E$ is twice continuously Frechét differentiable and $\psi: \mathbb{R} \times E \times[0,1] \rightarrow E$ is continuously Frechét differentiable with respect to $x, \varepsilon$ and $T$-periodic with respect to time. The functions $\phi$ and $\psi$ satisfy suitable condensivity conditions with respect to the Hausdorff measure of noncompactness. The crucial assumption is that the unperturbed equation at $\varepsilon=0$ has a continuous $T$-periodic isolated solution $x_{0}: \mathbb{R} \rightarrow E$, i.e., $x_{0} \in C_{T}(E)$.

The paper is organized as follows. In Section 2, we precise the conditions under which there is at least a branch of $T$-periodic solutions to (2) emanating from $x_{0}$. This existence result follows from the application of [36, Theorem 2]; this theorem relies on the method introduced in [23]. Precisely, to solve the bifurcation problem for (2), we introduce an equivalent integral equation whose zeros are the $T$-periodic solutions to (2) and that we rewrite in the following form:

$$
\tilde{P}(x)+\varepsilon \tilde{Q}(x, \varepsilon)=0,
$$

where $\tilde{P}: C_{T}(E) \rightarrow C_{T}(E)$ and $\tilde{Q}: C_{T}(E) \times[0,1] \rightarrow C_{T}(E)$. This equation has a branch of solutions originating from $x_{0}\left(\theta_{0}\right)$ if the Malkin bifurcation function given by

$$
M(\theta)=\int_{0}^{T}\left\langle\tilde{Q}\left(x_{0}(\theta), 0\right)(t), z_{0}(t+\theta)\right\rangle d t, \quad \theta \in[0, T]
$$


has $\theta_{0} \in[0, T]$ as simple zero. Here, for any $\theta \in[0, T], x_{0}(\theta) \in C_{T}(E)$ is given by $x_{0}(\theta)(\cdot):=$ $x_{\theta}(\cdot)=x(\cdot+\theta), z_{0}(\theta)$ is the eigenvector corresponding to the simple eigenvalue 0 of $\left(\tilde{P}^{\prime}\left(x_{0}(\theta)\right)\right)^{*}$ and $\langle\cdot, \cdot\rangle$ denotes the duality pairing of $E$ with its dual $E^{\prime}$. The main difficulty to verify the conditions of [36, Theorem 2] consists in proving that the zero eigenvalue of $\tilde{P}^{\prime}\left(x_{0}(\theta)\right)$ is simple. In fact, in this case, the assumption that the linearized equation, around the limit cycle $x_{0}(\theta), \theta \in[0, T]$, of the autonomous system at $\varepsilon=0$, does not have neither $T$-periodic solutions linearly independent with $x_{0}^{\prime}(\theta)$ nor Floquet adjoint solutions to $x_{0}^{\prime}(\theta)$ does not guarantee that the zero eigenvalue of $\tilde{P}^{\prime}\left(x_{0}(\theta)\right)$ is simple. To overcome this difficulty, we define in Section 3 a novel integral operator, equivalent to that associated to (2) with the property that for the resulting equation $P(x)+\varepsilon Q(x, \varepsilon)=0$, the operator $P^{\prime}\left(x_{0}(\theta)\right)$ has 0 as simple eigenvalue.

Furthermore, in Section 4, we calculate the Malkin bifurcation function associated to the integral operator introduced in Section 3, and we formulate in Theorem 2 the result of the existence of a branch of $T$-periodic solutions parameterized by $\varepsilon \geq 0$ small. Proposition 1 of Section 5 states a somewhat surprising result: the Malkin functions associated to the two integral operators coincide and they have the common form of the classical Malkin function introduced for ordinary differential equations in finite dimensional spaces of the form (1). Finally, in Section 6, we provide a concrete example of a system of partial differential equations to which our abstract bifurcation result applies.

\section{Assumptions and statement of the problem}

The paper deals with the problem of the existence of bifurcation of $T$-periodic solutions for the $T$-periodically perturbed autonomous equation of the form

$$
\dot{x}=A x+\phi(x)+\varepsilon \psi(t, x, \varepsilon), \quad t \geq 0, \varepsilon \geq 0,
$$

from a $T$-periodic limit cycle $x_{0}$ of the unperturbed system corresponding to $\varepsilon=0$. Here, $A$ is the infinitesimal generator of a strongly continuous semigroup $e^{A t}, t \geq 0$, acting in the Banach space $E$, which satisfies the Radon-Nikodym property; see [37, Theorem 23, p.276]; $\phi: E \rightarrow E, \psi: \mathbb{R} \times E \times[0,1] \rightarrow E$ is $T$-periodic and $x \in C_{T}(E)$, the space of $T$ periodic continuous functions $x: \mathbb{R} \rightarrow E$.

Throughout the paper, we assume the following conditions on $A, \phi$ and $\psi$.

(H1) $\left(e^{A t}\right)^{*}=e^{A^{*} t}$ and there exists $\alpha_{0}>0$ such that

$$
\left\|e^{A t}\right\|_{E} \leq e^{-\alpha_{0} t}, \quad t \geq 0
$$

(H2) $\phi$ is twice continuously Fréchet differentiable, $\psi$ is continuously Fréchet differentiable with respect to the pair $(x, \varepsilon)$. Moreover, for any nonempty, bounded set $\Omega \subset E$ we have

$$
\begin{aligned}
& \chi(\phi(\Omega)) \leq k \chi(\Omega) ; \\
& \chi(\psi([0, T] \times \Omega \times[0,1])) \leq l \chi(\Omega),
\end{aligned}
$$

where $0<k / \alpha_{0}<1, l>0$ and $\chi(\cdot)$ is the Hausdorff measure of noncompactness [38].

(H3) The unperturbed equation

$$
\dot{x}=A x+\phi(x)
$$


has a $T$-periodic isolated solution $x_{0} \in C_{T}(E)$, hence the set of shifts $x_{\theta}(\cdot)=x_{0}(\cdot+\theta)$, for any $\theta \in[0, T]$, represents a family of $T$-periodic solutions to (4). Moreover, for $\theta \in[0, T]$, we have that $y_{\theta}(t):=x_{\theta}^{\prime}(t)$ is a $T$-periodic solution to the linearized equation

$$
\dot{y}=A y+\phi^{\prime}\left(x_{0}(\theta)\right) y
$$

where $x_{0}(\theta):=x_{\theta}$. We assume that $x_{0}^{\prime}(\theta) \neq 0$ for any $\theta \in[0, T]$ and that (5) does not possess neither $T$-periodic solution linearly independent with $y_{\theta}(t)$ nor Floquet adjoint solution to $y_{\theta}(t)$, whenever $\theta \in[0, T]$, i.e., (5) does not have solutions of the form

$$
y(t)=v(t)+\frac{t}{T} y_{\theta}(t)
$$

where $v(t)$ is a $T$-periodic function.

We pose the following.

Problem To find conditions to ensure the existence of a branch of $T$-periodic solutions to (3) parameterized by $\varepsilon \geq 0$, originating, for some $\theta_{0} \in[0, T]$, from the family of $T$-periodic solutions $x_{0}(\theta)$.

To solve this problem, we first reduce the existence of $T$-periodic solutions to (3) to the problem of finding fixed points of an integral equation. For this, we introduce the linear operator $J: C_{T}(E) \rightarrow C_{T}(E)$ as follows:

$$
(J y)(t):=e^{A t}\left(I-e^{A T}\right)^{-1} \int_{0}^{T} e^{A(T-s)} y(s) d s+\int_{0}^{t} e^{A(t-s)} y(s) d s .
$$

Therefore, if we let

$$
\left(J r_{\varepsilon}(y)\right)(t):=J(\phi(y(t))+\varepsilon \psi(t, y(t), \varepsilon))
$$

then a function $y \in C_{T}(E)$ satisfying

$$
J\left(r_{\varepsilon}(y)\right)=y
$$

is a solution to (3) and vice versa. Moreover, it is easy to verify that the equation

$$
\left(J^{\prime}\left(r_{0}\left(x_{\theta}\right)\right)\right) y=y
$$

is equivalent to the linearized unperturbed equation

$$
\dot{y}=A y+a_{\theta}(t) y,
$$

where $a_{\theta}(t):=\phi^{\prime}\left(x_{0}(t+\theta)\right)$. Hence, we can rewrite (6) in the following form:

$$
\tilde{P}(y)+\varepsilon \tilde{Q}(y, \varepsilon)=0,
$$


where $\tilde{P}: C_{T}(E) \rightarrow C_{T}(E)$ and $\tilde{Q}: C_{T}(E) \times[0,1] \rightarrow C_{T}(E)$ are defined as follows:

$$
\tilde{P}(y)(t):=y(t)-e^{A t}\left(I-e^{A T}\right)^{-1} \int_{0}^{T} e^{A(T-s)} \phi(y(s)) d s-\int_{0}^{t} e^{A(t-s)} \phi(y(s)) d s,
$$

and

$$
\tilde{Q}(y, \varepsilon)(t):=-e^{A t}\left(I-e^{A T}\right)^{-1} \int_{0}^{T} e^{A(T-s)} \psi(s, y(s), \varepsilon) d s-\int_{0}^{t} e^{A(t-s)} \psi(s, y(s), \varepsilon) d s .
$$

In conclusion, our problem will be solved if we show that for $\varepsilon \geq 0$ sufficiently small, equation (7) has a solution. To this end, it would be sufficient to verify the conditions of the following result.

Theorem 1 ([36, Theorem 2]) Let $B$ be a Banach space, let $P: B \rightarrow B$ be a twice continuously Fréchet differentiable map and $Q: B \times[0,1] \rightarrow B$ continuously Fréchet differentiable with respect to both the variables.

Assume that the equation $P(x)=0$ has one-dimensional set of solutions $x_{0}(\theta) \in B$, parameterized by $\theta \in[0, T]$, such that there exists $x_{0}^{\prime \prime}(\theta)$ for any $\theta \in[0, T]$ and $x_{0}^{\prime}(\theta) \neq 0$ for any $\theta \in[0, T]$. Assume that the eigenvalue $0 \in \sigma\left(P^{\prime}\left(x_{0}(\theta)\right)\right)$ is simple and the operator $I-P^{\prime}\left(x_{0}(\theta)\right): B \rightarrow B$ is compact, whenever $\theta \in[0, T]$. Consider the function defined by

$$
M(\theta):=\int_{0}^{T}\left\langle Q\left(x_{0}(\theta), 0\right)(t), z_{0}(t+\theta)\right\rangle d t
$$

where $z_{0}(\theta)$ is the eigenvector corresponding to the simple eigenvalue $0 \in \sigma\left(P^{\prime}\left(x_{0}(\theta)\right)\right)^{*}$. Here, $*$ denotes the adjoint operator.

Then, for each $\theta_{0} \in[0, T]$ such that $M\left(\theta_{0}\right)=0$ and $M^{\prime}\left(\theta_{0}\right) \neq 0$ the equation $P(x)+$ $\varepsilon Q(x, \varepsilon)=0$ is solvable, for $\varepsilon \geq 0$ sufficiently small, in a neighborhood of the point $x_{0}\left(\theta_{0}\right)$ and the solution has the form

$$
x(\varepsilon)=x_{0}\left(\theta_{0}\right)+\varepsilon w+o(\varepsilon)
$$

where $w \in B$ can be determined in explicit form as shown in [35, Theorem 2.3] and [36, Lemma 3].

As it has been observed in [39], the compactness of the operator $I-P^{\prime}\left(x_{0}(\theta)\right)$ can be replaced by the condensivity of $I-P^{\prime}\left(x_{0}(\theta)\right)$ with respect to the Hausdorff measure of noncompactness. Indeed, as it is shown in [40], under assumptions (H1)-(H2), the operator $I-P$ and thus $I-P^{\prime}\left(x_{0}(\theta)\right)$, see [38, Theorem 1.5.4], are condensing with constant $k / \alpha_{0}<1$. Furthermore, [38, Theorem 2.6.11] ensures that zero is an eigenvalue of $P^{\prime}\left(x_{0}(\theta)\right)$ of finite multiplicity. Therefore, under assumptions (H1)-(H3), one can easily verify that the conditions of the previous Theorem 1 are satisfied for (7) except the condition of the simplicity of the zero eigenvalue of $\tilde{P}^{\prime}\left(x_{0}(\theta)\right), \theta \in[0, T]$. In fact, the assumption that (5) does not possess neither $T$-periodic solutions linearly independent with $y_{\theta}(t)$, nor Floquet adjoint solutions to $y_{\theta}(t)$, whenever $\theta \in[0, T]$, does not imply that the zero eigenvalue of $\tilde{P}^{\prime}\left(x_{0}(\theta)\right)$ is simple, despite the existence of a bijection between the $T$-periodic solutions to (5) and 
the $T$-periodic solutions to $\tilde{P}^{\prime}\left(x_{0}(\theta)\right)=0$. Moreover, as it shown in [41] the simplicity of the zero eigenvalue of $\tilde{P}^{\prime}\left(x_{0}(\theta)\right)$ does not imply that the $T$-periodic solution $y_{\theta}(t)$ to $(5)$ has the property mentioned above.

In conclusion, in order to apply Theorem 1, we will introduce a novel integral operator whose fixed points are also fixed points of (6) and vice versa, and thus $T$-periodic solutions to (3). Moreover, we will show that the zero eigenvalue of the corresponding operator $P^{\prime}\left(x_{0}(\theta)\right)$ is simple. This is the aim of the next section.

\section{A novel equivalent integral operator}

Let $F_{\varepsilon}(y):=\tilde{P}(y)+\varepsilon \tilde{Q}(y, \varepsilon)$, hence equation (7) reads as $F_{\varepsilon}(y)=0$. Consider the integral equation

$$
F_{\varepsilon}(y)(t)-\xi(t) \int_{0}^{\tau} F_{\varepsilon}(y)(s) d s=0, \quad t \in[0, T]
$$

where $\xi: E \rightarrow C_{T}(E)$ is defined in the next lemma and $\tau \in[0, T]$ is a given point.

For any fixed $\theta \in[0, T]$, let $e_{0}:=x_{0}^{\prime}(\theta) \neq 0, \gamma_{0}:=\int_{0}^{\tau} e_{0}(s) d s$ and $\beta:=\left\langle f, \int_{0}^{\tau}\left(J e_{0}\right)(s) d s\right\rangle$ where $f \in E^{\prime}$.

We can now formulate the following result.

Lemma 1 Assume $(\mathrm{H} 1)-(\mathrm{H} 3)$ and that $\tau \in[0, T]$ and $f \in E^{\prime}$ satisfy the conditions:

(H4) $\gamma_{0} \neq 0$.

(H5) $\beta \neq 0$.

(H6) $\left\langle f, \gamma_{0}\right\rangle=1$.

Define $\xi: E \rightarrow C_{T}(E)$ as follows:

$$
\forall x \in E, \quad \xi(t) x:=\langle f, x\rangle g(t), \quad t \in[0, T]
$$

where $g:=e_{0}+\frac{1}{\beta}\left(J e_{0}\right) \in C_{T}(E)$. Then (8) is equivalent to (6). Moreover, the zero eigenvalue of $P^{\prime}\left(x_{0}(\theta)\right)$ is simple, where $P: C_{T}(E) \rightarrow C_{T}(E)$ is given by

$$
P(y)(t):=\tilde{P}(y)(t)-\xi(t) \int_{0}^{\tau} \tilde{P}(y)(s) d s, \quad t \in[0, T]
$$

Proof First of all observe that, under our assumptions, we have that

$$
1 \notin \sigma\left(\int_{0}^{\tau} \xi(s) d s\right)
$$

Indeed, arguing by contradiction assume that $\hat{y} \in E$ is such that

$$
\left(\int_{0}^{\tau} \xi(s) d s\right) \hat{y}=\hat{y}
$$

then, by the definition of $\xi$, we obtain

$$
\left(\int_{0}^{\tau} \xi(s) d s\right) \hat{y}=\langle f, \hat{y}\rangle \int_{0}^{\tau} g(s) d s
$$


hence

$$
\hat{y}=\langle f, \hat{y}\rangle \int_{0}^{\tau} g(s) d s
$$

thus $\hat{y}$ and $\int_{0}^{\tau} g(s) d s$ are linearly dependent, i.e., $\int_{0}^{\tau} g(s) d s$ is also an eigenvector of $\int_{0}^{\tau} \xi(s) d s$, that is,

$$
\int_{0}^{\tau} \xi(s) d s \int_{0}^{\tau} g(s) d s=\int_{0}^{\tau} g(s) d s
$$

In conclusion, we should have

$$
\left\langle f, \int_{0}^{\tau} g(s) d s\right\rangle=1
$$

On the other hand, as it is easy to verify our conditions imply that

$$
\left\langle f, \int_{0}^{\tau} g(s) d s\right\rangle=2
$$

We now prove the equivalence between (6) and (8). Clearly, if $F_{\varepsilon}(y)=0$ for some $y \in$ $C_{T}(E)$ then (8) is satisfied. Conversely, assume that $y \in C_{T}(E)$ is a solution to (8), hence

$$
F_{\varepsilon}(y)(t)-\xi(t) \int_{0}^{\tau} F_{\varepsilon}(y)(s) d s=0, \quad t \in[0, T]
$$

Integrating on the interval $[0, \tau]$, we obtain

$$
\int_{0}^{\tau} F_{\varepsilon}(y)(s) d s-\int_{0}^{\tau} \xi(s) d s \int_{0}^{\tau} F_{\varepsilon}(y)(s) d s=0,
$$

or equivalently,

$$
\left(I-\int_{0}^{\tau} \xi(s) d s\right) \int_{0}^{\tau} F_{\varepsilon}(y)(s) d s=0 .
$$

Since $1 \notin \sigma\left(\int_{0}^{\tau} \xi(s) d s\right)$ from (10), it follows that $\int_{0}^{\tau} F_{\varepsilon}(y)(s) d s=0$ and so from (8) we get $F_{\varepsilon}(y)=0$.

It remains to prove the second part of the lemma. For this, given $\theta \in[0, T]$, let $a_{\theta}(t):=$ $\phi^{\prime}\left(x_{0}(t+\theta)\right), t \in[0, T]$, and let $\left(\alpha_{\theta} y\right)(t):=a_{\theta}(t) y(t)$. To simplify the notation in the sequel, we omit the subscript $\theta$. Observe that

$$
F_{0}^{\prime}\left(x_{0}(\theta)\right) y=\tilde{P}^{\prime}\left(x_{0}(\theta)\right) y=y-J(\alpha y)
$$

Then the equation

$$
\left(F_{0}^{\prime}\left(x_{0}(\theta)\right) y\right)(t)-\xi(t) \int_{0}^{\tau}\left(F_{0}^{\prime}\left(x_{0}(\theta)\right) y\right)(s) d s=0
$$


can be rewritten as follows:

$$
y(t)-(J(\alpha y))(t)-\xi(t) \int_{0}^{\tau}(y(s)-(J(\alpha y))(s)) d s=0 .
$$

For $t \in[0, T]$, define

$$
P(y)(t):=\tilde{P}(y)(t)-\xi(t) \int_{0}^{\tau} \tilde{P}(y)(s) d s
$$

and

$$
Q(y, \varepsilon)(t):=\tilde{Q}(y, \varepsilon)(t)-\xi(t) \int_{0}^{\tau} \tilde{Q}(y, \varepsilon)(s) d s
$$

then the equation (11) takes the form

$$
P^{\prime}\left(x_{0}(\theta)\right) y=0 \text {. }
$$

Clearly, $e_{0}$ is an eigenvector of $P^{\prime}\left(x_{0}(\theta)\right)$ corresponding to the zero eigenvalue, i.e., $P^{\prime}\left(x_{0}(\theta)\right) e_{0}=0$. Assume now that there exists an adjoint vector $e_{1}$ to $e_{0}$, namely

$$
P^{\prime}\left(x_{0}(\theta)\right) e_{1}=e_{0}
$$

or

$$
e_{1}(t)-\left(J\left(\alpha e_{1}\right)\right)(t)-\xi(t) \int_{0}^{\tau}\left(e_{1}(s)-\left(J\left(\alpha e_{1}\right)\right)(s)\right) d s=e_{0}(t)
$$

for any $t \in[0, T]$. By assumption, there are no adjoint Floquet solutions to (5), thus

$$
\dot{e}_{1}(t)=A e_{1}(t)+a(t) e_{1}(t)-e_{0}(t)
$$

does not possess $T$-periodic solution $e_{1}(t)$. The integral form of (13) is given by

$$
e_{1}(t)=\left(J\left(\alpha e_{1}\right)\right)(t)-\left(J e_{0}\right)(t)
$$

Therefore, it remains to show that (12) and (14) coincide, namely

$$
\xi(t) \int_{0}^{\tau}\left(e_{1}(s)-\left(J\left(\alpha e_{1}\right)\right)(s)\right) d s+e_{0}(t)=-\left(J e_{0}\right)(t) .
$$

For this, integrating (12) on the interval $[0, \tau]$, we obtain

$$
\int_{0}^{\tau}\left(e_{1}(s)-\left(J\left(\alpha e_{1}\right)\right)(s)\right) d s-\int_{0}^{\tau} \xi(s) d s \int_{0}^{\tau}\left(e_{1}(s)-\left(J\left(\alpha e_{1}\right)\right)(s)\right) d s=\int_{0}^{\tau} e_{0}(s) d s,
$$

that is

$$
\left(I-\int_{0}^{\tau} \xi(s) d s\right) \int_{0}^{\tau}\left(e_{1}(s)-\left(J\left(\alpha e_{1}\right)\right)(s)\right) d s=\int_{0}^{\tau} e_{0}(s) d s
$$


On the other hand $1 \notin \sigma\left(\int_{0}^{\tau} \xi(s) d s\right)$, thus

$$
\int_{0}^{\tau}\left(e_{1}(s)-\left(J\left(\alpha e_{1}\right)\right)(s)\right) d s=\left(I-\int_{0}^{\tau} \xi(s) d s\right)^{-1} \int_{0}^{\tau} e_{0}(s) d s .
$$

From (16), we get the following form for (15)

$$
\xi(t)\left(I-\int_{0}^{\tau} \xi(s) d s\right)^{-1} \int_{0}^{\tau} e_{0}(s) d s=-e_{0}(t)-\left(J e_{0}\right)(t) .
$$

Then

$$
\left(I-\int_{0}^{\tau} \xi(s) d s-I\right)\left(I-\int_{0}^{\tau} \xi(s) d s\right)^{-1} \int_{0}^{\tau} e_{0}(s) d s=\int_{0}^{\tau}\left(e_{0}(s)+\left(J e_{0}\right)(s)\right) d s,
$$

hence

$$
\int_{0}^{\tau} e_{0}(s) d s-\left(I-\int_{0}^{\tau} \xi(s) d s\right)^{-1} \int_{0}^{\tau} e_{0}(s) d s=\int_{0}^{\tau}\left(e_{0}(s)+\left(J e_{0}\right)(s)\right) d s,
$$

and

$$
\left(I-\int_{0}^{\tau} \xi(s) d s\right)^{-1} \int_{0}^{\tau} e_{0}(s) d s=-\int_{0}^{\tau}\left(J e_{0}\right)(s) d s
$$

Finally substituting (18) into (17), we obtain

$$
\xi(t) \int_{0}^{\tau}\left(J e_{0}\right)(s) d s=e_{0}(t)+\left(J e_{0}\right)(t) .
$$

By our definition $\xi(t) x=\langle f, x\rangle g(t), g(t)=\frac{1}{\beta}\left(e_{0}(t)+\left(J e_{0}\right)(t)\right)$, with $\left\langle f, \int_{0}^{\tau} e_{0}(s) d s\right\rangle=1$ and $\left\langle f, \int_{0}^{\tau}\left(J e_{0}\right)(s) d s\right\rangle=\beta$. Therefore, (19) is satisfied and this concludes the proof.

Remark 1 Observe that a little though convinces of the existence of $\tau \in[0, T]$ and $f \in E^{\prime}$ satisfying the conditions (H4)-(H6) of Lemma 1.

Furthermore, recall that assumptions (H1)-(H2) ensure that the operator $I-P$ is condensing with constant $k / \alpha_{0}<1$ (see [40]); moreover, [38, Theorem 1.5.4] guarantees that $I-P^{\prime}\left(x_{0}(\theta)\right)$ is also condensing with the same constant. Finally, by [38, Theorem 2.6.11], zero turns out to be an eigenvalue of $P^{\prime}\left(x_{0}(\theta)\right)$ of finite multiplicity. The second part of the proof of Lemma 1 shows that it is simple.

\section{The Malkin bifurcation function}

In the previous section, Lemma 1 states that the operator $P$, associated to the integral equation (8) satisfies the conditions of Theorem 1 . This section is devoted to the computation of the following Malkin bifurcation function $M_{\xi}(\theta)$ associated to (8)

$$
M_{\xi}(\theta):=\int_{0}^{T}\left\langle\tilde{Q}\left(x_{0}(\theta), 0\right)(t), z_{0}(t+\theta)\right\rangle d t-\int_{0}^{T}\left\langle\xi(t) \int_{0}^{\tau} \tilde{Q}\left(x_{0}(\theta), 0\right)(s) d s, z_{0}(t+\theta)\right\rangle d t
$$


where $z_{0}(\theta)$ is an eigenvector of $\left(P^{\prime}\left(x_{0}(\theta)\right)\right)^{*}$, i.e.,

$$
\left(P^{\prime}\left(x_{0}(\theta)\right)\right)^{*} z_{0}(\theta)=0 \text {. }
$$

For notational convenience, we simply denote $z_{0}(\theta) \in C_{T}(E)$ by $z_{0}$. In order to compute $M_{\xi}(\theta)$, it is necessary to determine $z_{0}$ in explicit form. The following result solves the problem.

Lemma 2 Assume (H1)-(H6), we have that

$$
z_{0}(t)=\gamma(t)-\mathbb{1}_{[0, \tau]}(t) \int_{0}^{T}\langle g(t), \gamma(t)\rangle f d t
$$

where $\gamma$ is an eigenvector of $(J \alpha)^{*}$ corresponding to the eigenvalue 1 and $\mathbb{1}_{[0, \tau]}$ is the characteristic function of the interval $[0, \tau]$.

Proof By assumption, $E$ has the Radon-Nikodym property, then the eigenvalue of $\left(P^{\prime}\left(x_{0}(\theta)\right)\right)^{*}$ can be determined, without loss of generality in the Hilbert space $H_{T}^{1}$. Hence, for any $x, y \in C_{T}(E)$ we have

$$
\begin{aligned}
& \int_{0}^{T}\left\langle P^{\prime}\left(x_{0}(\theta)\right) x(t), y(t)\right\rangle d t \\
& \quad=\int_{0}^{T}\left\langle x(t)-(J(\alpha x))(t)-\xi(t) \int_{0}^{\tau}(x(s)-(J(\alpha x))(s)) d s, y(t)\right\rangle d t .
\end{aligned}
$$

First, by using assumption (H1), we calculate

$$
\begin{aligned}
\int_{0}^{T} & \langle(J(\alpha x))(t), y(t)\rangle d t \\
= & \int_{0}^{T}\left\langle e^{A t}\left(I-e^{A T}\right)^{-1} \int_{0}^{T} e^{A(T-s)} a(s) x(s) d s+\int_{0}^{t} e^{A(t-s)} a(s) x(s) d s, y(t)\right\rangle d t \\
= & \int_{0}^{T}\left\langle\int_{0}^{T} e^{A(T-s)} a(s) x(s) d s,\left(I-e^{A^{*} T}\right)^{-1} e^{A^{*} t} y(t)\right\rangle d t \\
& +\int_{0}^{T}\left\langle\int_{0}^{t} e^{A(t-s)} a(s) x(s) d s, y(t)\right\rangle d t \\
= & \int_{0}^{T} \int_{0}^{T}\left\langle e^{A(T-s)} a(s) x(s),\left(I-e^{A^{*} T}\right)^{-1} e^{A^{*} t} y(t)\right\rangle d s d t \\
& +\int_{0}^{T} \int_{0}^{t}\left\langle e^{A(t-s)} a(s) x(s), y(t)\right\rangle d s d t \\
= & \int_{0}^{T} \int_{0}^{T}\left\langle e^{-A s} a(s) x(s), e^{A^{*} T}\left(I-e^{A^{*} T}\right)^{-1} e^{A^{*} t} y(t)\right\rangle d t d s \\
& +\int_{0}^{T} \int_{s}^{T}\left\langle e^{-A s} a(s) x(s), e^{A^{*} t} y(t)\right\rangle d t d s \\
= & \int_{0}^{T}\left\langle x(s), \int_{0}^{T} a^{*}(s) e^{A^{*}(T-s)}\left(I-e^{A^{*} T}\right)^{-1} e^{A^{*} t} y(t) d t\right\rangle d s \\
& +\int_{0}^{T}\left\langle x(s), \int_{s}^{T} a *(s) e^{A^{*}(t-s)} y(t) d t\right\rangle d s .
\end{aligned}
$$


Therefore, we have

$$
\left((J \alpha)^{*} y\right)(t)=\int_{0}^{T} a^{*}(t) e^{A^{*}(T-t)}\left(I-e^{A^{*} T}\right)^{-1} e^{A^{*} s} y(s) d s+\int_{t}^{T} a^{*}(t) e^{A^{*}(s-t)} y(s) d s .
$$

We now calculate the adjoint operator for $\xi \int_{0}^{\tau}(x(s)-(J(\alpha x))(s)) d s$. For this, consider

$$
\begin{aligned}
\int_{0}^{T}\left\langle\xi(t) \int_{0}^{\tau}(x(s)-(J(\alpha x))(s)) d s, y(t)\right\rangle d t \\
\quad=\int_{0}^{T}\left\langle\int_{0}^{\tau}(x(s)-(J(\alpha x))(s)) d s, \xi^{*}(t) y(t)\right\rangle d t \\
=\int_{0}^{T}\left\langle\int_{0}^{T} \mathbb{1}_{[0, \tau]}(s)(x(s)-(J(\alpha x))(s)) d s, \xi^{*}(t) y(t)\right\rangle d t \\
=\int_{0}^{T}\left\langle\mathbb{1}_{[0, \tau]}(s)(x(s)-(J(\alpha x))(s)), \int_{0}^{T} \xi^{*}(t) y(t) d t\right\rangle d s \\
=\int_{0}^{T}\left\langle x(s)-(J(\alpha x))(s), \mathbb{1}_{[0, \tau]}(s) \int_{0}^{T} \xi^{*}(t) y(t) d t\right\rangle d s \\
=\int_{0}^{T}\left\langle x(s), \mathbb{1}_{[0, \tau]}(s) \int_{0}^{T} \xi^{*}(t) y(t) d t\right\rangle d s-\int_{0}^{T}\left\langle x(s),(J \alpha)^{*} \mathbb{1}_{[0, \tau]}(s) \int_{0}^{T} \xi^{*}(t) y(t) d t\right\rangle d s \\
=\int_{0}^{T}\left\langle x(s), \mathbb{1}_{[0, \tau]}(s) \int_{0}^{T} \xi^{*}(t) y(t) d t-(J \alpha)^{*} \mathbb{1}_{[0, \tau]}(s) \int_{0}^{T} \xi^{*}(t) y(t) d t\right\rangle d s .
\end{aligned}
$$

Thus,

$$
\begin{aligned}
& \left(\xi(t) \int_{0}^{\tau}\left(y(s)-\left((J \alpha)^{*} y\right)(s)\right) d s\right)^{*} \\
& \quad=\mathbb{1}_{[0, \tau]}(t) \int_{0}^{T} \xi^{*}(t) y(t) d t-(J \alpha)^{*} \mathbb{1}_{[0, \tau]}(t) \int_{0}^{T} \xi^{*}(t) y(t) d t .
\end{aligned}
$$

Finally, we calculate $\xi^{*}(t)$, for this consider

$$
\langle\xi(t) x, y\rangle=\langle\langle f, x\rangle g(t), y\rangle=\langle f, x\rangle\langle g(t), y\rangle=\langle x,\langle g(t), y\rangle f\rangle,
$$

i.e.

$$
\xi^{*}(t) y=\langle g(t), y\rangle f
$$

Now, we are in the position to determine the eigenvector $z_{0}$ of $\left(P^{\prime}\left(x_{0}(\theta)\right)\right)^{*}$. We have that

$$
z_{0}(t)=(J \alpha)^{*} z_{0}(t)+\mathbb{1}_{[0, \tau]}(t) \int_{0}^{T}\left\langle g(s), z_{0}(s)\right| f d s-(J \alpha)^{*} \mathbb{1}_{[0, \tau]}(t) \int_{0}^{T}\left\langle g(s), z_{0}(s)\right| f d s
$$

Then

$$
z_{0}(t)-\mathbb{1}_{[0, \tau]}(t) \int_{0}^{T}\left\langle g(s), z_{0}(s)\right| f d s=(J \alpha)^{*}\left(z_{0}(t)-\mathbb{1}_{[0, \tau]}(t) \int_{0}^{T}\left\langle g(s), z_{0}(s)\right\rangle f d s\right) .
$$


Let $\gamma(t):=z_{0}(t)-\mathbb{1}_{[0, \tau]}(t) \int_{0}^{T}\left\langle g(s), z_{0}(s)\right\rangle f d s$, then the previous equation takes the form

$$
\gamma=(J \alpha)^{*} \gamma
$$

namely $\gamma$ is an eigenvector of the linear operator $(J \alpha)^{*}: C_{T}(E) \rightarrow C_{T}(E)$ corresponding to the simple eigenvalue 1 . Therefore, by replacing $y$ with $\gamma$ in (20), we obtain

$$
\gamma(t)=\int_{0}^{T} a^{*}(t) e^{A^{*}(T-t)}\left(I-e^{A^{*} T}\right)^{-1} e^{A^{*} s} \gamma(s) d s+\int_{t}^{T} a^{*}(t) e^{A^{*}(s-t)} \gamma(s) d s .
$$

The aim now is to find $\gamma$, for this consider the adjoint equation to (5)

$$
\dot{v}(t)=-A^{*} v(t)-a^{*}(t) v(t)
$$

and the solution of (22), defined for $t \in[0, T]$, given by

$$
v(t)=e^{-A^{*}(t-T)} v(T)+\int_{T}^{t} e^{-A^{*}(t-s)}\left(-a^{*}(s)\right) v(s) d s .
$$

For $t=0$, we have

$$
v(0)=e^{A^{*} T} v(T)+\int_{0}^{T} e^{A^{*} s} a^{*}(s) v(s) d s .
$$

Since $v$ is $T$-periodic, we obtain

$$
v(T)=\left(I-e^{A^{*} T}\right)^{-1} \int_{0}^{T} e^{A^{*} s} a^{*}(s) v(s) d s .
$$

By using (24) into (23), we get

$$
v(t)=e^{A^{*}(T-t)}\left(I-e^{A^{*} T}\right)^{-1} \int_{0}^{T} e^{A^{*} s} a^{*}(s) v(s) d s+\int_{t}^{T} e^{-A^{*}(t-s)} a^{*}(s) v(s) d s .
$$

Put $\omega(t):=-\dot{v}(t)-A^{*} v(t)$, then $\omega(t)=a^{*}(t) v(t)$ and (25) becomes

$$
\nu(t)=e^{A^{*}(T-t)}\left(I-e^{A^{*} T}\right)^{-1} \int_{0}^{T} e^{A^{*} s} \omega(s) d s+\int_{t}^{T} e^{A^{*}(s-t)} \omega(s) d s .
$$

Therefore,

$$
\omega(t)=\int_{0}^{T} a^{*}(t) e^{A^{*}(T-t)}\left(I-e^{A^{*} T}\right)^{-1} e^{A^{*} s} \omega(s) d s+\int_{t}^{T} a^{*}(t) e^{A^{*}(s-t)} \omega(s) d s,
$$

i.e., $\omega(t)$ is a solution to (21). Hence,

$$
\gamma(t)=-\dot{v}(t)-A^{*} v(t)
$$

where $v(t)$ is the $T$-periodic solution to the adjoint equation (22). Finally, from

$$
z_{0}(t)-\mathbb{1}_{[0, \tau]}(t) \int_{0}^{T}\left\langle g(s), z_{0}(s)\right| f d s=\gamma(t),
$$


we obtain

$$
\begin{aligned}
& \int_{0}^{T}\left\langle g(t), z_{0}(t)\right\rangle d t-\int_{0}^{T} \mathbb{1}_{[0, \tau]}(t) \int_{0}^{T}\left\langle g(s), z_{0}(s)\right\rangle d s|g(t), f\rangle d t \\
& \quad=\int_{0}^{T}\langle g(t), \gamma(t)\rangle d t
\end{aligned}
$$

and

$$
\int_{0}^{T}\left\langle g(t), z_{0}(t)\right\rangle d t-\int_{0}^{T}\left\langle g(s), z_{0}(s)\right\rangle d s\left\langle\int_{0}^{\tau} g(t) d t, f\right\rangle=\int_{0}^{T}\langle g(t), \gamma(t)\rangle d t .
$$

By (9), we get

$$
\int_{0}^{T}\left\langle g(t), z_{0}(t)\right\rangle d t=-\int_{0}^{T}\langle g(t), \gamma(t)\rangle d t
$$

In conclusion,

$$
z_{0}(t)=\gamma(t)-\mathbb{1}_{[0, \tau]}(t) \int_{0}^{T}\langle g(t), \gamma(t)\rangle f d t .
$$

Lemmas 1 and 2, together with the fact that (H1)-(H2) ensure the condensivity of $I-$ $P^{\prime}\left(x_{0}(\theta)\right), \theta \in[0, T]$, of constant $0<k / \alpha_{0}<1$ (see [40]) allow to apply Theorem 1 to state the following.

Theorem 2 Assume (H1)-(H6). If there exists $\theta_{0} \in[0, T]$ such that $M_{\xi}\left(\theta_{0}\right)=0$ and $M_{\xi}^{\prime}\left(\theta_{0}\right) \neq 0$. Then there exists a branch of $T$-periodic solutions to (3) of the form

$$
x(\varepsilon)=x_{0}\left(\theta_{0}\right)+\varepsilon w+o(\varepsilon)
$$

for $\varepsilon \geq 0$ sufficiently small and $w \in C_{T}(E)$.

Remark 2 The function $w$ can be calculated in an explicit form as shown in [35, Theorem 2.3] and [36, Lemma 3].

\section{An invariance property of the Malkin bifurcation function}

In what follows, we state an interesting property of the Malkin bifurcation functions introduced before. Precisely, we can prove the following result.

Proposition 1 Let $\theta \in[0, T]$, assume that $0 \in \sigma\left(\tilde{P}^{\prime}\left(x_{0}(\theta)\right)\right)$ is simple. Then the Malkin bifurcation function $M(\theta)$ associated to system (6) coincide with the Malkin bifurcation function $M_{\xi}(\theta)$ associated to system (8).

Proof Consider

$$
\begin{aligned}
M_{\xi}(\theta) & =\int_{0}^{T}\left\langle\tilde{Q}\left(x_{0}(\theta), 0\right)(t), z_{0}(t)\right\rangle d t-\int_{0}^{T}\left\langle\xi(t) \int_{0}^{\tau} \tilde{Q}\left(x_{0}(\theta), 0\right)(s) d s, z_{0}(t)\right\rangle d t \\
& =\int_{0}^{T}\left\langle\tilde{Q}\left(x_{0}(\theta), 0\right)(t), \gamma(t)\right\rangle d t
\end{aligned}
$$




$$
\begin{aligned}
& -\int_{0}^{T}\left\langle\tilde{Q}\left(x_{0}(\theta), 0\right)(t), \mathbb{1}_{[0, \tau]}(t) \int_{0}^{T}\langle g(s), \gamma(s)\rangle d s f\right\rangle d t \\
& -\int_{0}^{T}\left\langle\xi(t) \int_{0}^{\tau} \tilde{Q}\left(x_{0}(\theta), 0\right)(s) d s, \gamma(t)-\mathbb{1}_{[0, \tau]}(t) \int_{0}^{T}\langle g(s), \gamma(s)\rangle d s f\right\rangle d t .
\end{aligned}
$$

Let $\mu:=\int_{0}^{T}\langle g(s), \gamma(s)\rangle d s$, then by (9) we have

$$
\begin{aligned}
M_{\xi}(\theta)= & \int_{0}^{T}\left\langle\tilde{Q}\left(x_{0}(\theta), 0\right)(t), \gamma(t)\right\rangle d t-\int_{0}^{T}\left\langle\tilde{Q}\left(x_{0}(\theta), 0\right)(t), \mathbb{1}_{[0, \tau]}(t) \mu f\right\rangle d t \\
& -\int_{0}^{T}\left\langle\left\langle f, \int_{0}^{\tau} \tilde{Q}\left(x_{0}(\theta), 0\right)(s) d s\right\rangle g(t), \gamma(t)-\mathbb{1}_{[0, \tau]}(t) \mu f\right\rangle d t \\
= & \int_{0}^{T}\left\langle\tilde{Q}\left(x_{0}(\theta), 0\right)(t), \gamma(t)\right\rangle d t-\int_{0}^{T}\left\langle\tilde{Q}\left(x_{0}(\theta), 0\right)(t), \mathbb{1}_{[0, \tau]}(t) \mu f\right\rangle d t \\
& -\int_{0}^{T}\left\langle\left\langle f, \int_{0}^{\tau} \tilde{Q}\left(x_{0}(\theta), 0\right)(s) d s\right\rangle g(t), \gamma(t)\right\rangle d t \\
& +\int_{0}^{T}\left\langle\left\langle f, \int_{0}^{\tau} \tilde{Q}\left(x_{0}(\theta), 0\right)(s) d s\right\rangle g(t), \mathbb{1}_{[0, \tau]}(t) \mu f\right\rangle d t \\
= & \int_{0}^{T}\left\langle\tilde{Q}\left(x_{0}(\theta), 0\right)(t), \gamma(t)\right\rangle d t-2 \int_{0}^{T}\left\langle\tilde{Q}\left(x_{0}(\theta), 0\right)(t), \mathbb{1}_{[0, \tau]}(t) \mu f\right\rangle d t \\
& +\int_{0}^{T}\left\langle f, \int_{0}^{\tau} \tilde{Q}\left(x_{0}(\theta), 0\right)(s) d s\right\rangle\left\langle g(t), \mathbb{1}_{[0, \tau]}(t) \mu f\right\rangle d t \\
= & \int_{0}^{T}\left\langle\tilde{Q}\left(x_{0}(\theta), 0\right)(t), \gamma(t)\right\rangle d t-2 \int_{0}^{\tau}\left\langle\tilde{Q}\left(x_{0}(\theta), 0\right)(t), \mu f\right\rangle d t \\
& +2 \mu\left\langle f, \int_{0}^{\tau} \tilde{Q}\left(x_{0}(\theta), 0\right)(s) d s\right\rangle=\int_{0}^{T}\left\langle\tilde{Q}\left(x_{0}(\theta), 0\right)(t), \gamma(t)\right\rangle d t=M(\theta) .
\end{aligned}
$$

Remark $3 M(\theta)$ can be rewritten in the classical form of the Malkin bifurcation function $f_{0}(\theta)$ for ordinary differential equations as $(1)$ of the Introduction. In fact, consider

$$
\begin{aligned}
M(\theta)= & \int_{0}^{T}\left\langle\tilde{Q}\left(x_{0}(\theta), 0\right)(t), \gamma(t)\right\rangle d t=\int_{0}^{T}\left\langle-\left(J \Psi\left(x_{0}(\theta)\right)\right)(t),-\dot{v}(t)-A^{*} v(t)\right\rangle d t \\
= & \int_{0}^{T}\left\langle\left(J \Psi\left(x_{0}(\theta)\right)\right)(s), \dot{v}(s)\right\rangle d s+\int_{0}^{T}\left\langle\left(J \Psi\left(x_{0}(\theta)\right)\right)(s), A^{*} v(s)\right\rangle d s \\
= & \left.\left\langle\left(J \Psi\left(x_{0}(\theta)\right)\right)(t), v(t)\right\rangle\right|_{0} ^{T}-\int_{0}^{T}\left\langle A\left(J \Psi\left(x_{0}(\theta)\right)\right)(t)+\Psi\left(x_{0}(\theta)\right)(t), v(t)\right\rangle d t \\
& +\int_{0}^{T} A\left\langle\left(J \Psi\left(x_{0}(\theta)\right)\right)(t), v(t)\right\rangle d t .
\end{aligned}
$$

Since $\left(J \Psi\left(x_{0}(\theta)\right)\right)(0)=\left(J \Psi\left(x_{0}(\theta)\right)\right)(T), v(0)=v(T), \Psi$ is the superposition operator generated by $\psi$ and $v$ solves (22) we have

$$
M(\theta)=\int_{0}^{T}\left\langle\psi\left(t, x_{0}(t+\theta), 0\right), v(t+\theta)\right\rangle d t .
$$




\section{An example}

In order to introduce an example consistent with the general approach of the paper that requires the employ of the theory of condensing operators, we are led to consider partial differential equations of hyperbolic type, whose abstract formulation in Banach spaces gives rise to infinitesimal generators of noncompact $C_{0}$-semigroups; see, e.g., [42].

Precisely, following [43] and [44], we present a concrete, not academic example concerning the existence of periodic solutions of a system of two autonomous damped wave equations in a thin domain with Neumann boundary conditions. The study of the dynamics of partial differential equations in thin domains has received many attention in the past few years; see [45] and the extensive references therein. The system has the form

$$
\begin{aligned}
& \frac{\partial^{2} u_{1}}{\partial t^{2}}=\Delta_{x} u_{1}+\frac{1}{\lambda^{2}} \frac{\partial^{2} u_{1}}{\partial y^{2}}-\beta_{1} \frac{\partial u_{1}}{\partial t}-\alpha_{1} u_{1}+\phi_{1}\left(x, \lambda y, u_{1}, u_{2}\right), \\
& \frac{\partial^{2} u_{2}}{\partial t^{2}}=\Delta_{x} u_{2}+\frac{1}{\lambda^{2}} \frac{\partial^{2} u_{2}}{\partial y^{2}}-\beta_{2} \frac{\partial u_{2}}{\partial t}-\alpha_{2} u_{2}+\phi_{2}\left(x, \lambda y, u_{1}, u_{2}\right), \\
& \frac{\partial u_{1}}{\partial v}=\frac{\partial u_{2}}{\partial v}=0
\end{aligned}
$$

where $(x, y) \in Q:=\Omega \times(0,1) \subset \mathbb{R}^{n+1}, n \geq 2, \Omega$ is a $C^{2}$-smooth bounded domain in $\mathbb{R}^{n}, v$ denotes the outward unit normal vector to $Q, \lambda$ is a small positive parameter representing the thickness of the domain of the variable $\lambda y, \alpha_{1}, \alpha_{2}, \beta_{1}, \beta_{2}$ are positive constants and the functions $\phi_{1}, \phi_{2}$ are of class $C^{1}$ jointly in their arguments. The linear part of system (26) generates an exponentially stable $C_{0}$-semigroup in a suitable Banach space; see [43] as well as the related references therein. Under the assumption of the existence of a $T_{0}$-periodic solution $u_{0}=\left(u_{1}^{0}, u_{2}^{0}\right)$ of the limit problem, obtained as $\lambda \rightarrow 0$, and suitable conditions on the growth of the derivatives of $\phi_{1}, \phi_{2}$ with respect to their arguments, it is shown in [44] that [43, Theorem 1] applies. This result guarantees the existence of $\lambda_{0}>0$ such that, for fixed $\hat{\lambda} \in\left(0, \lambda_{0}\right)$, system (26) has an isolated $T_{\hat{\lambda}}$-periodic solution $u_{\hat{\lambda}}=\left(u_{1}^{\hat{\lambda}}, u_{2}^{\hat{\lambda}}\right)$. The crucial assumption of [43, Theorem 1$]$ is that the zero eigenvalue of the linearized system around $u_{0}$ is simple. For a single damped wave equation of system (26) with the nonlinear term $\phi$ depending periodically on time $t$, the existence of periodic solutions was studied in [46].

Consider now a $T_{\hat{\lambda}}$-periodic perturbation of (26) of small amplitude $\varepsilon>0$

$$
\begin{aligned}
\frac{\partial^{2} u_{1}}{\partial t^{2}}= & \Delta_{x} u_{1}+\frac{1}{\hat{\lambda}^{2}} \frac{\partial^{2} u_{1}}{\partial y^{2}}-\beta_{1} \frac{\partial u_{1}}{\partial t}-\alpha_{1} u_{1}+\phi_{1}\left(x, \hat{\lambda} y, u_{1}, u_{2}\right) \\
& +\varepsilon \psi_{1}\left(\varepsilon, t, x, \hat{\lambda} y, u_{1}, u_{2}\right), \\
\frac{\partial^{2} u_{2}}{\partial t^{2}}= & \Delta_{x} u_{2}+\frac{1}{\hat{\lambda}^{2}} \frac{\partial^{2} u_{2}}{\partial y^{2}}-\beta_{2} \frac{\partial u_{2}}{\partial t}-\alpha_{2} u_{2}+\phi_{2}\left(x, \hat{\lambda} y, u_{1}, u_{2}\right) \\
& +\varepsilon \psi_{2}\left(\varepsilon, t, x, \hat{\lambda} y, u_{1}, u_{2}\right), \\
\frac{\partial u_{1}}{\partial v}= & \frac{\partial u_{2}}{\partial v}=0 .
\end{aligned}
$$

If we assume that the superposition operators generated by the functions $\phi_{1}, \phi_{2}, \psi_{1}, \psi_{2}$ satisfy assumption (H2) of this paper, then (H1) and [47, Theorem 4.3.1] ensure that the $C_{0}$-semigroup generated by the linearization around $u_{\hat{\lambda}}$ of the unperturbed system, corresponding to $\varepsilon=0$ in (27), is strongly contractive with respect to the Hausdorff measure 
of noncompactness $\chi(\cdot)$, i.e., $\chi$-strongly contractive. Therefore, our abstract bifurcation result Theorem 2 applies to system (27).

\section{Competing interests}

The authors declare that they have no competing interests.

\section{Authors' contributions}

The authors wrote this paper in collaboration and with the same responsibility. All authors read and approved the final version of the manuscript.

\section{Author details}

${ }^{1}$ Department of Mathematics, Voronezh State University, Voronezh, 394006, Russia. ²Dipartimento di Ingegneria dell'Informazione e Scienze Matematiche, Università di Siena, Siena, 53100, Italy.

\section{Acknowledgements}

Dedicated to professor Jean Mawhin on the occasion of his seventieth birthday. The first two authors acknowledge the support by RFBR Grants 10-01-93112-a and 12-01-0392-a. The third one acknowledges the support by the GNAMPA of the Istituto di Alta Matematica. The authors would like also to thank the referees for their helpful comments and suggestions which improved the presentation of the paper.

\section{Received: 24 December 2012 Accepted: 6 April 2013 Published: 23 April 2013}

\section{References}

1. Awrejcewicz, J, Lamarque, C-H: Bifurcation and Chaos in Nonsmooth Mechanical System. World Scientific, Singapore (2003)

2. Awrejcewicz, J, Holicke, MM: Smooth and Nonsmooth High Dimensional Chaos and the Melnikov-Type Methods. World Scientific, Singapore (2007)

3. di Bernardo, M, Budd, CJ, Champneys, AR, Kowalczyk, P: Piecewise-Smooth Dynamical Systems, Theory and Applications. Springer, London (2008)

4. Fečkan, M: Topological Degree Approach to Bifurcation Problems. Springer, Berlin (2008)

5. Fečkan, M: Bifurcation and Chaos in Discontinuous and Continuous Systems. Springer, Berlin (2011)

6. di Bernardo, M, Budd, CJ, Champneys, AR, Kowalczyk, P, Nordmark, AB, Olivar, G, Piiroinen, PT: Bifurcations in nonsmooth dynamical systems. SIAM Rev. 50, 629-701 (2008)

7. Mawhin, J: Topological bifurcation theory: old and new. In: Progress in Variational Methods. Nankai Ser. Pure Appl. Math. Theoret. Phys., vol. 7, pp. 169-186. World Scientific, Hackensak (2011)

8. Loud, WS: Periodic solutions of a perturbed autonomous system. Ann. Math. 70, 490-529 (1959)

9. Malkin, IG: On Poincaré's theory of periodic solutions. Akad. Nauk SSSR. Prikl. Mat. Meh. 13, 633-646 (1949) (in Russian)

10. Melnikov, VK: On the stability of a center for time-periodic perturbations. Tr. Mosk. Mat. Obŝ. 12, 3-52 (1963) (in Russian)

11. Rhouma, MBH, Chicone, C: On the continuation of periodic orbits. Methods Appl. Anal. 7, 85-104 (2000)

12. Hausrath, AR, Manásevich, RF: Periodic solutions of periodically harvested Lotka-Volterra systems. Rev. Colomb. Mat. 21, 337-345 (1987)

13. Hausrath, AR, Manásevich, RF: Periodic solutions of periodically forced nondegenerate systems. Rocky Mt. J. Math. 18 49-65 (1988)

14. Hausrath, AR, Manásevich, RF: Stability properties of periodic solutions of periodically forced non degenerate systems. Rocky Mt. J. Math. 18, 785-800 (1988)

15. Ortega, R: A criterion for asymptotic stability based on topological degree. In: Proceedings of the First World Congress of Nonlinear Analysts, Tampa, FL, pp. 383-394 (1992)

16. Blekhman, II: Synchronization in Nature and Engineering. Nauka, Moscow (1981) (in Russian)

17. Guckenheimer, J, Holmes, P: Nonlinear Oscillations, Dynamical Systems, and Bifurcations of Vector Fields. Applied Mathematical Sciences, vol. 42. Springer, New York (1983)

18. Greenspan, B, Holmes, P: Repeated resonance and homoclinic bifurcation in a periodically forced family of oscillators SIAM J. Math. Anal. 15, 69-97 (1984)

19. Lazer, AC: Small periodic perturbations of a class of conservative systems. J. Differ. Equ. 13, 438-456 (1973)

20. Tkhai, VN: Periodic motions of a system close to an autonomous reversible system. J. Appl. Math. Mech. 65, 647-664 (2001)

21. Palmer, KJ: Exponential dichotomies and transversal homoclinic points. J. Differ. Equ. 55, 225-256 (1984)

22. Battelli, F, Fečkan, M: Some remarks on the Melnikov function. Electron. J. Differ. Equ. 2002, 1-29 (2002)

23. Kamenskii, M, Makarenkov, O, Nistri, P: An alternative approach to study bifurcation from a limit cycle in periodically perturbed autonomous systems. J. Dyn. Differ. Equ. 23, 425-435 (2011). doi:10.1007/s10884-011-9207-4

24. Kamenskii, M, Mikhaylenko, B, Nistri, P: Nonsmooth bifurcation in finite dimensional spaces via scaling of variables. Differ. Equ. Dyn. Syst. 20, 191-205 (2012)

25. Mawhin, J: Topological Degree Methods in Nonlinear Boundary Value Problems. CBMS Reg. Conf. Ser. Math., vol. 40. Am. Math. Soc., Providence (1979)

26. Mawhin, J: Leray-Schauder continuation theorems in the absence of a priori bounds. Topol. Methods Nonlinear Anal. 9, 179-200 (1997)

27. Mawhin, J: Periodic solutions in the golden sixties: the birth of a continuation theorem. In: Ten Mathematical Essays on Approximation in Analysis and Topology, pp. 199-214. Elsevier, Amsterdam (2005)

28. Capietto, A, Mawhin, J, Zanolin, F: Continuation theorems for periodic perturbations of autonomous systems. Trans. Am. Math. Soc. 329, 41-72 (1992) 
29. Henrard, M, Zanolin, F: Bifurcation from a periodic orbit in perturbed planar Hamiltonian systems. J. Math. Anal. Appl. 277, 79-103 (2003)

30. Kamenskii, M, Makarenkov, O, Nistri, P: A continuation principle for a class of periodically perturbed autonomous systems. Math. Nachr. 281, 42-61 (2008)

31. Makarenkov, O, Nistri, P: Periodic solutions for planar autonomous systems with nonsmooth periodic perturbations. J. Math. Anal. Appl. 338, 42-61 (2008)

32. Kamenskii, M, Makarenkov, O, Nistri, P: Small parameter perturbations of nonlinear periodic systems. Nonlinearity 17, 193-205 (2004)

33. Makarenkov, O, Nistri, $\mathrm{P}:$ On the rate of convergence of periodic solutions in perturbed autonomous systems as the perturbation vanishes. Commun. Pure Appl. Anal. 17, 49-61 (2008)

34. Makarenkov, O, Malaguti, L, Nistri, P: On the behavior of periodic solutions of planar autonomous Hamiltonian systems with multivalued periodic perturbations. Z. Anal. Anwend. 30, 129-144 (2011)

35. Couchouron, JF, Kamenski, M, Nistri, P: An infinite dimensional bifurcation problem with application to a class of functional differential equations of neutral type. Commun. Pure Appl. Anal. 12, 1845-1859 (2013) doi:10.3934/cpaa.2013.12.1845

36. Kamenskii, MI, Mikhaylenko, BA: Averaging principle and the variational approach in the problem on the bifurcation of periodic solutions from non-isolated equilibria of the averaged equation. Differ. Equ. 48, 1-8 (2012)

37. Royden, HL: Real Analysis, 3rd edn. Macmillan Co., New York (1988)

38. Akhmerov, RR, Kamenski, MI, Potapov, AS, Rodkina, AN, Sadovskii, BN: Measures of Noncompactness and Condensing Operators. Operator Theory: Advances and Applications, vol. 55. Birkhäuser, Basel (1992)

39. Kamenskii, MI, Mikhaylenko, BA: On perturbations of systems with multidimensional degeneration. Autom. Remote Control 72, 1036-1047 (2011)

40. Kamenskii, M, Nistri, P: An averaging method for singularly perturbed systems of semi linear differential inclusions with Co-semigroups. Set-Valued Anal. 11, 345-357 (2003)

41. Mikhaylenko, BA: On equivalent integral operators in periodic solutions problems of differential equations. Proc. Voronezh State Univ. Voronezh 1, 193-201 (2011) (in Russian)

42. Pazy, A: Semigroups of Linear Operator and Applications to Partial Differential Equations. Applied Mathematical Sciences, vol. 44. Springer, New York (1983)

43. Johnson, R, Kamenskii, M, Nistri, P: Existence of periodic solutions for an autonomous damped wave equation in a thin domain. J. Dyn. Differ. Equ. 10, 409-424 (1998)

44. Johnson, R, Kamenskii, M, Nistri, P: Erratum to existence of periodic solutions for an autonomous damped wave equation in a thin domain. J. Dyn. Differ. Equ. 12, 675-679 (2000)

45. Raugel, G: Dynamics of Partial Differential Equations in Thin Domains. Lecture Notes in Mathematics, vol. 1609, pp. 208-315. Springer, Berlin (1995)

46. Johnson, R, Kamenskii, M, Nistri, P: On periodic solutions of a damped wave equation in a thin domain using degree theoretic methods. J. Differ. Equ. 140, 186-208 (1997)

47. Kamenskii, M, Obukhovskii, V, Zecca, P: Condensing Multivalued Maps and Semilinear Differential Inclusions in Banach Spaces. De Gruyter Series in Nonlinear Analysis and Applications. de Gruyter, Berlin (2001)

doi:10.1186/1687-2770-2013-101

Cite this article as: Kamenskii et al.: A bifurcation problem for a class of periodically perturbed autonomous parabolic equations. Boundary Value Problems 2013 2013:101.

\section{Submit your manuscript to a SpringerOpen ${ }^{\circ}$ journal and benefit from:}

- Convenient online submission

Rigorous peer review

- Immediate publication on acceptance

- Open access: articles freely available online

- High visibility within the field

- Retaining the copyright to your article 